\title{
Calculation of Coefficients of Cassegrain Telescope Mirrors
}

\author{
Ahmed Kamil Ahmed * \\ Received 16, February, 2009 \\ Accepted 25, May, 2009
}

\begin{abstract}
:
In the present work, a program for calculating the coefficients of the Aplanatic Cassegrain Telescope (ACT) system, free from the effects of spherical and coma aberrations, were constructed. In addition, the two-mirrors of the optical system, as aspherical surfaces, were adopted. This means, that the two-equations of the mirrors are assumed to be polynomial function of five even terms only. The numerical method, least-squares curve fitting method to calculate the two-mirror coefficients system, was adopted. For choosing the values and ratios that give the best results, Rayleigh Criterion (Rayleigh Limit), for purpose of comparison and preference, was adopted.
\end{abstract}

Key words: Telescope, Aplanatic Cassegrain Telescope, Coefficients, Primary Mirror, Secondary Mirror, Aspherical Surfaces.

\section{Introduction:}

A telescope is an instrument designed for the observation of remote objects by the collection of electromagnetic radiation. The most important type of reflecting telescope is Cassegrain type [1]. A reflecting telescope, or reflector, is one in which the objective is a mirror [2]. The mirror is close to the rear of the telescope and light is bounced off (reflected) as it strikes the mirror. Cassegrain telescope $^{1}$ gives a longer focal length and higher magnification [3].

Cassegrain telescope has twocurved reflecting mirror. The parabolic primary concave mirror has a hole at its center, and is placed at the bottom of the telescope tube. A smaller hyperbole secondary convex mirror is placed near the top of the telescope. The secondary mirror bounces the light from the primary mirror back down the tube through the hole in the primary, to the focus point behind [3], as shown in Figure (1):

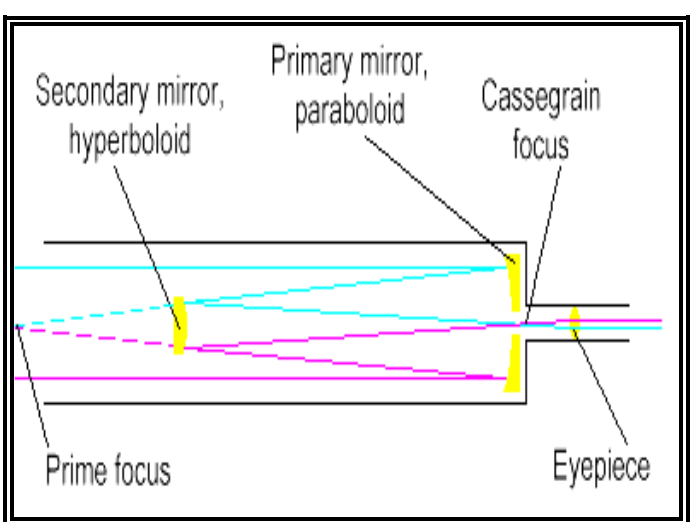

Fig. (1): Cassegrain telescope optical layout [3]

\section{Aplanatism:}

The term aplanatism was used to imply axial stigmatism together with the satisfaction of the exact sine condition $^{2}$ [5]. A spherical surface whose position is relative to an object point (either real or virtual), the rays are refracted through the surface free from all orders of spherical and linear coma aberrations are called an aplanatic surface.

\footnotetext{
${ }^{1}$ :The French astronomer Gaillaume Cassegrain in 1672 invented this type of telescopes [4].

${ }^{2}$ Abbe and Helmholtz discovered the sine theorem almost simultaneously but quite independently in 1873 [9].

*Department of Astronomy - College of Science - University of Baghdad
} 
Any surface is aplanatic with respect to one particular object point. This point and its image

conjugate are known as the aplanatic points $^{2}$ of the surface [6].

\section{Aspherical Surfaces:}

Aspherical surfaces, or "aspherics", are optical surfaces that are neither spherical nor plane, used in imaging and non-imaging systems [7]. Optical designers have used the following aspheric formula [8]:

$$
x=\frac{c r^{2}}{1+\sqrt{1-(K+1) c^{2} r^{2}}}+a_{1} r^{4}+a_{2} r^{6}+a_{3} r^{8}+a_{4} r^{10}+\cdots
$$

Where $x$ coordinate of this surface is given as a power series expansion in $r$, where:

$$
r=\sqrt{y^{2}+z^{2}} \ldots(2)
$$

Equation (1) represents the aspheric as surface of revolution about the x-axis. Figure (2), whose base curve is a conic section, with vertex curvature $c$ and conic constant $k$ [11].

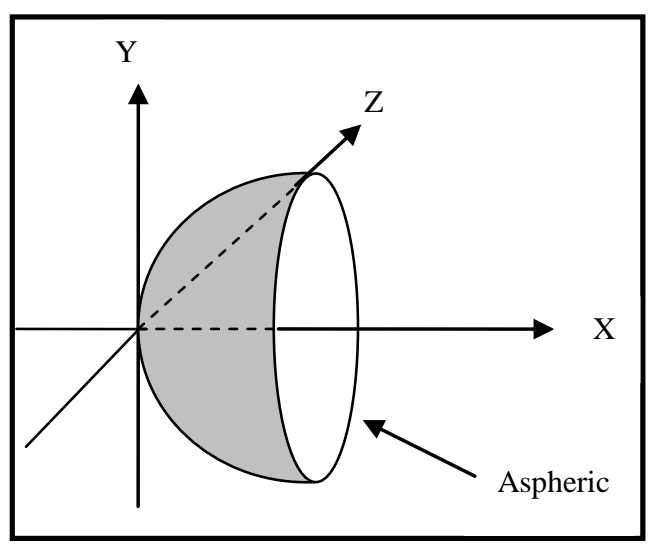

Fig. (2), Aspheric surface [5]

The first term in equation (1) represents the conic sections and the other terms represent the deformations from this conic section. Other forms of representation have also been used as [12]:

$$
x=a_{0} r^{2}+a_{1} r^{4}+a_{2} r^{6}+a_{3} r^{8}+a_{4} r^{10}+\cdots(3)
$$

The design of aspherical system raises a number of questions. For most among these are the problems of how to determine for any given system the aspheric profiles so that to obtain a good definition over the whole of a finite field [13].

The aim of present work is to provide a method, for calculating the coefficients of the Aplanatic Cassegrain Telescope (ACT) system, free from the effects of spherical and coma aberrations.

\section{Profile Determination of the Optical System:}

We exhibit the main formulas is used for calculating the two-mirror profiles. Figure (3), shows the mirrors profile of the Aplanatic Cassegrain Telescope (ACT) system [1].

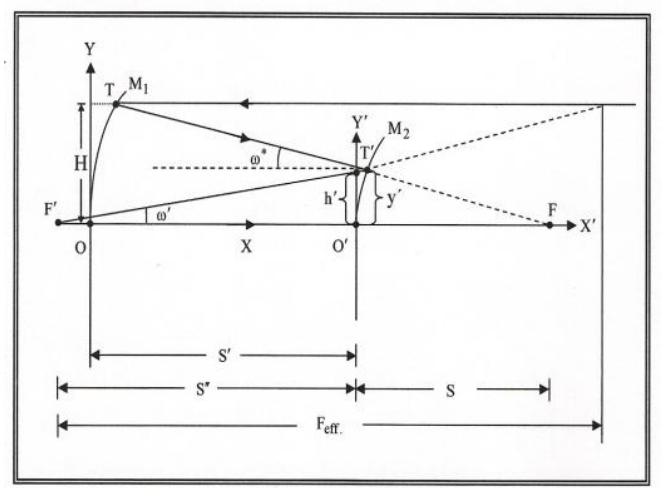

Fig. (3): The mirrors profile of the Aplanatic Cassegrain Telescope (ACT) system [1]

The equation of the primary mirror profile is given by [1]:

$\frac{d h^{\prime}}{d H}=\frac{S^{\prime \prime} H^{2}}{\left[{F_{\text {eff. }}}^{2}-H^{2}\right]^{\frac{3}{2}}}+\frac{S^{\prime \prime}}{\left[{F_{\text {eff. }}}^{2}-H^{2}\right]^{\frac{1}{2}}}$

The equation of the secondary mirror profile is given by [1]:

$$
\frac{d X^{\prime}}{d H}=\frac{\left[\frac{d h^{\prime}}{d H}+X^{\prime} \frac{d \operatorname{Tan}\left(\omega^{\prime}\right)}{d H}\right] \operatorname{Tan}\left(\frac{\omega^{*}-\omega^{\prime}}{2}\right)}{1-\operatorname{Tan}\left(\omega^{\prime}\right) \operatorname{Tan}\left(\frac{\omega^{*}-\omega^{\prime}}{2}\right)}
$$

\footnotetext{
${ }^{1}$ In general a spherical surface does not form a perfect image of a point. There are, however, a few points that are imaged without aberrations, these called aplanatic points [10].
} 
Symbols in previous equations, are as follows [1]:

$h^{\prime}$ : Height of the ray from $\mathrm{x}$-axis of the secondary mirror.

$H$ : Height of the ray from $\mathrm{x}$-axis of the primary mirror.

$S^{\prime \prime}$ : Distance of the secondary mirror from the final focal plane $\left(F^{\prime} O^{\prime}\right)$.

$\omega^{*}$ : Angle between the reflected ray from the primary mirror and the $\mathrm{x}$-axis. $\omega^{\prime}$ : Angle between the reflected ray from the secondary mirror and the $\mathrm{x}$ axis.

$F_{\text {eff: }}$ : Effective focal length of the optical system.

\section{Numerical Method:}

The numerical method used to calculate the coefficients of the mirrors, for the optical system, is the least-squares curve fitting method. It is the equation of a smooth curve, which does not pass through each of a number of given points, but which passes near each of them in a plane. The "nearness" is usually obtained by imposing the least-squares criterions, and the application of this criterion is the basis of the method of least squares.
The problem of fitting a set of data with a polynomial of $m$ th degree in the form [14]:

$Y_{i}=k_{0}+k_{1} x_{i}+k_{2} x_{i}^{2}+k_{3} x_{i}^{3}+\cdots+k_{m} x_{i}^{m} \ldots(6)$
We next determine the coefficients $k_{0}$ and $k_{1}$ by using the least-squares criterions which requires that $S=\sum_{i=1}^{n}\left(Y_{i}-y_{i}\right)^{2}$ be a minimum, where $Y_{i}$ is evaluated from equation (6). The values $Y_{i}-y_{i}$ are called residuals.

Basically, we wish to find a minimum value of $S$, where:

$$
\begin{aligned}
& S=\sum_{i=1}^{n}\left(Y_{i}-y_{i}\right)^{2}= \\
& \sum_{i=1}^{n}\left(k_{0}+k_{1} x_{i}+k_{2} x_{i}^{2}+k_{3} x_{i}^{3}+\cdots+k_{m} x_{i}^{m}-y_{i}\right)^{2}
\end{aligned}
$$

Equation (7) modified to the form:

$$
\begin{aligned}
& S=\sum_{i=1}^{n}\left(Y_{i}-y_{i}\right)^{2}= \\
& \sum_{i=1}^{n}\left(k_{2} x_{i}^{2}+k_{4} x_{i}^{4}+k_{6} x_{i}^{6}+\cdots+k_{m} x_{i}^{m}-y_{i}\right)^{2}
\end{aligned}
$$

To obtain the minimum value of $S$, which is now a function of $m+2$ variables $k_{2}, k_{4}, k_{6}, k_{8}, k_{10}, \cdots, k_{m}$ we set the following $m+2$ first partial derivative to zero:

$$
\left.\begin{array}{l}
\frac{\partial S}{\partial k_{2}}=2 \sum_{i=1}^{n}\left(k_{2} x_{i}^{2}+k_{4} x_{i}^{4}+k_{6} x_{i}^{6}+k_{8} x_{i}^{8}+k_{10} x_{i}^{10}+\cdots+k_{m} x_{i}^{m}-y_{i}\right) x_{i}^{2}=0.0 \\
\frac{\partial S}{\partial k_{4}}=2 \sum_{i=1}^{n}\left(k_{2} x_{i}^{2}+k_{4} x_{i}^{4}+k_{6} x_{i}^{6}+k_{8} x_{i}^{8}+k_{10} x_{i}^{10}+\cdots+k_{m} x_{i}^{m}-y_{i}\right) x_{i}^{4}=0.0 \\
\frac{\partial S}{\partial k_{6}}=2 \sum_{i=1}^{n}\left(k_{2} x_{i}^{2}+k_{4} x_{i}^{4}+k_{6} x_{i}^{6}+k_{8} x_{i}^{8}+k_{10} x_{i}^{10}+\cdots+k_{m} x_{i}^{m}-y_{i}\right) x_{i}^{6}=0.0 \\
\frac{\partial S}{\partial k_{8}}=2 \sum_{i=1}^{n}\left(k_{2} x_{i}^{2}+k_{4} x_{i}^{4}+k_{6} x_{i}^{6}+k_{8} x_{i}^{8}+k_{10} x_{i}^{10}+\cdots+k_{m} x_{i}^{m}-y_{i}\right) x_{i}^{8}=0.0 \\
\frac{\partial S}{\partial k_{10}}=2 \sum_{i=1}^{n}\left(k_{2} x_{i}^{2}+k_{4} x_{i}^{4}+k_{6} x_{i}^{6}+k_{8} x_{i}^{8}+k_{10} x_{i}^{10}+\cdots+k_{m} x_{i}^{m}-y_{i}\right) x_{i}^{10}=0.0 \\
\vdots \\
\frac{\partial S}{\partial k_{m}}=2 \sum_{i=1}^{n}\left(k_{2} x_{i}^{2}+k_{4} x_{i}^{4}+k_{6} x_{i}^{6}+k_{8} x_{i}^{8}+k_{10} x_{i}^{10}+\cdots+k_{m} x_{i}^{m}-y_{i}\right) x_{i}^{m}=0.0
\end{array}\right\}
$$

We then obtain the $m+2$ simultaneous linear, or normal, equations: 


$$
\left.\begin{array}{l}
k_{2} \sum x_{i}^{4}+k_{4} \sum x_{i}^{6}+k_{6} \sum x_{i}^{8}+\cdots+k_{m} \sum x_{i}^{m+2}-\sum x_{i}^{2} y_{i}=0.0 \\
k_{2} \sum x_{i}^{6}+k_{4} \sum x_{i}^{8}+k_{6} \sum x_{i}^{10}+\cdots+k_{m} \sum x_{i}^{m+4}-\sum x_{i}^{4} y_{i}=0.0 \\
k_{2} \sum x_{i}^{8}+k_{4} \sum x_{i}^{10}+k_{6} \sum x_{i}^{12}+\cdots+k_{m} \sum x_{i}^{m+6}-\sum x_{i}^{6} y_{i}=0 \\
\vdots \\
k_{2} \sum x_{i}^{m+2}+k_{4} \sum x_{i}^{m+4}+k_{6} \sum x_{i}^{m+6}+\cdots+k_{m} \sum x_{i}^{m+m}-\sum x_{i}^{m} y_{i}=0.0
\end{array}\right\}
$$

Where the symbol $\sum$ implies summation for $i$ from 1 to $n$.

It is convenient to express to equation (10) in the following matrix notation [14].

$[A][k]=[B]$,

Where:

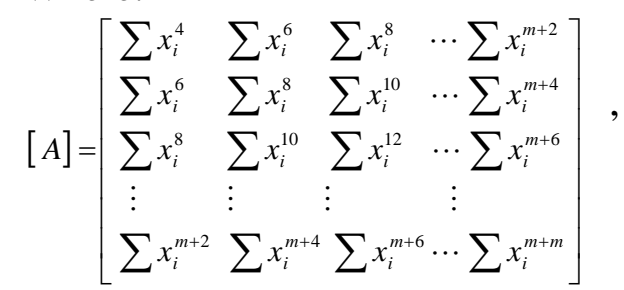

is a symmetrical matrix, and

$$
[k]=\left[\begin{array}{c}
k_{2} \\
k_{4} \\
k_{6} \\
\vdots \\
k_{m}
\end{array}\right], \quad[B]=\left[\begin{array}{c}
\sum x_{i}^{2} y_{i} \\
\sum x_{i}^{4} y_{i} \\
\sum x_{i}^{6} y_{i} \\
\vdots \\
\sum x_{i}^{m} y_{i}
\end{array}\right]
$$

\section{Results and Discussion:}

This section, displays the obtained results, from the program that have been constructed. In addition, the analysis and discussion completely dependent on conditions that made. Before giving the results, the principles that were taken into account are as follows [1]:

1- Primary Mirror Diameter $\left(D_{1}\right)$ : We choose three different dimensions of $\mathrm{D}_{1}=40,50$, and $60 \mathrm{~cm}$.

2- Focal Ratio of the Primary Mirror $\left(f_{1} / D_{1}\right)$ : We choose two different values of $f_{1} / D_{1}$ ratio, these ratios are $=$ 4 , and 7.

3- Obscuration Ratio of the Secondary Mirror $\left(D_{2} / D_{1}\right)$ : We choose one value of $D_{2} / D_{1}$ ratio, this ratio is $=$
0.3, which is calculated from the following equation [15]:

$\frac{D_{2}}{D_{1}} \geq \frac{S}{f_{1}}$

4- Separated Distance Between TwoMirrors (S'), Distance of the Secondary Mirror From the Primary Focus (S), and Effective Focal Length of the Optical System $\left(F_{\text {eff. }}\right)$ : Different values of $\mathrm{S}^{\prime}, \mathrm{S}$, and $\mathrm{F}_{\text {eff. have been }}$ adopted, according to the following equation [15]:

$$
F_{\text {eff. }}=\frac{S^{\prime}}{S} f_{1}
$$

\section{5- Ratio of the Effective Focal Length} to the Primary Mirror Diameter $\left(F_{\text {eff. }} /\right.$ $\left.D_{1}\right)$ or $\quad(F$-number $)$ or $(F / N o$.$) :$ Different values of $F / N o$. ratio have been adopted from the previous points, according to the following equation [15].

$$
\frac{F_{\text {eff. }}}{D_{1}}=\frac{S^{\prime}}{S} \frac{f_{1}}{D_{1}}=F \text {-number or F/No... }
$$

Table (1); tabulate the calculated values [1].

Table (1): Values of the calculated parameters, for $\mathrm{D}_{2} / \mathrm{D}_{1}=0.3[1]$

\begin{tabular}{|c|c|c|c|c|c|c|}
\hline $\begin{array}{c}\mathrm{D}_{1} \\
(\mathrm{~cm})\end{array}$ & $\mathrm{f}_{1} / \mathrm{D}_{1}$ & $\begin{array}{c}\mathrm{f}_{1} \\
(\mathrm{~cm})\end{array}$ & $\begin{array}{c}\mathrm{S} \\
(\mathrm{cm})\end{array}$ & $\begin{array}{c}\mathrm{S}^{\prime} \\
(\mathrm{cm})\end{array}$ & $\begin{array}{c}\mathrm{F}_{\text {eff. }} \\
(\mathrm{cm})\end{array}$ & F/No. \\
\hline \multirow{2}{*}{40} & 4 & 160 & 48 & 112 & 373.33 & 9.33 \\
\cline { 2 - 7 } & 7 & 280 & 84 & 196 & 653.33 & 16.33 \\
\hline \multirow{2}{*}{50} & 4 & 200 & 60 & 140 & 466.67 & 7.47 \\
\cline { 2 - 7 } & 7 & 350 & 105 & 245 & 816.67 & 13.07 \\
\hline \multirow{2}{*}{60} & 4 & 240 & 72 & 168 & 559.99 & 6.22 \\
\cline { 2 - 7 } & 7 & 420 & 126 & 294 & 979.99 & 10.89 \\
\hline
\end{tabular}

Now, we display the flowchart for mirrors coefficients calculation, as shown in Figure (4). 


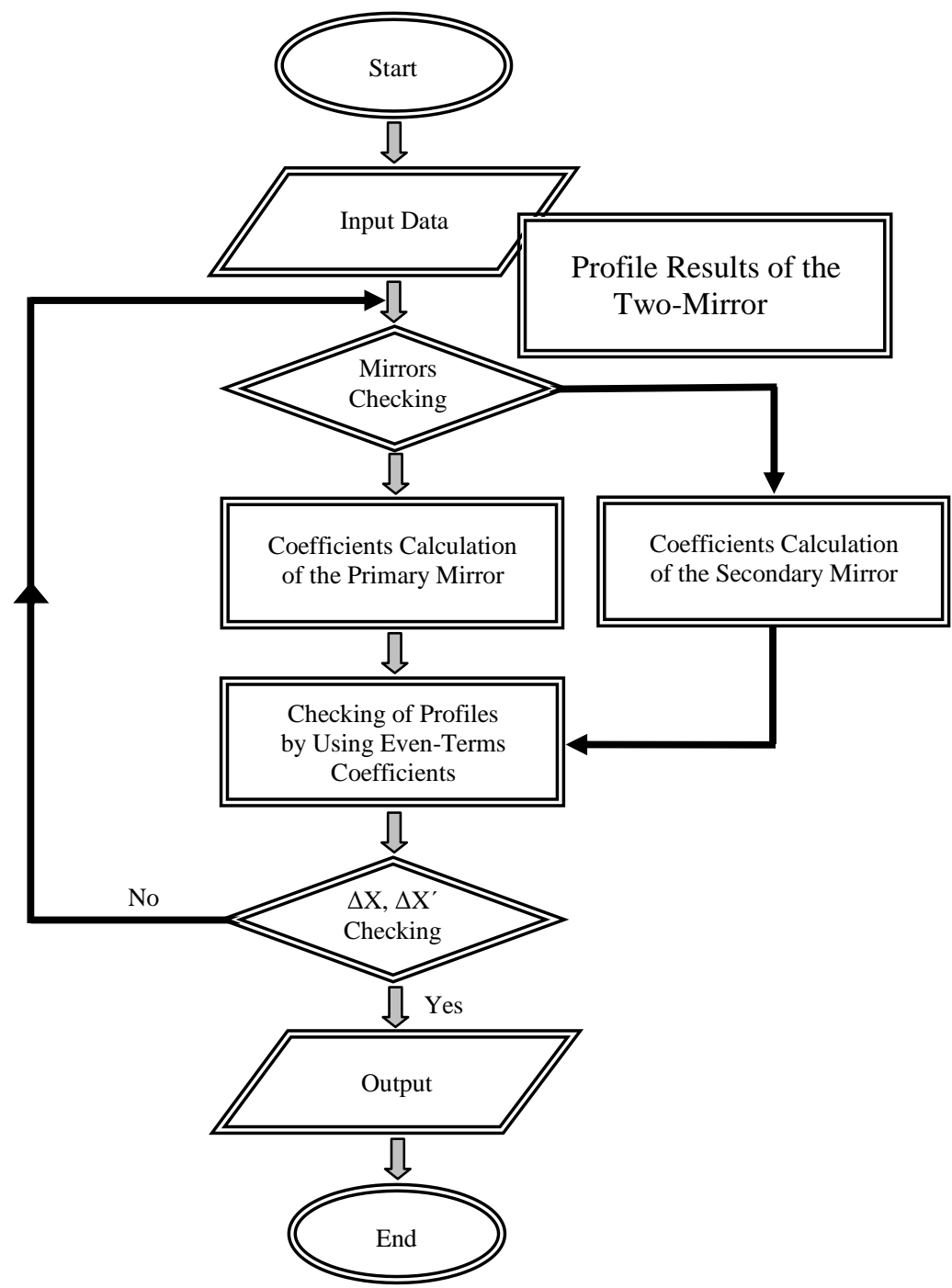

Fig. (4): Flowchart for the two-mirror coefficients calculation

Tables (2) and (3), shows the results of the two-mirror coefficients, for $\mathrm{D}_{1}=$ 40,50 , and $60 \mathrm{~cm}, \mathrm{f}_{1} / \mathrm{D}_{1}=4,7$ and $\mathrm{D}_{2} / \mathrm{D}_{1}=0.3$

Table (2): Coefficients results of the mirrors, for $f_{1} / D_{1}=4, D_{2} / D_{1}=0.3$, and different values of $D_{1}$

\begin{tabular}{|c|c|c|}
\hline $\mathbf{D}_{\mathbf{1}}(\mathbf{c m})$ & Primary Mirror & Secondary Mirror \\
\hline \multirow{4}{*}{40} & $0.1564 \mathrm{E}-02$ & $0.2976 \mathrm{E}-02$ \\
\cline { 2 - 3 } & $-0.2525 \mathrm{E}-07$ & $-0.2282 \mathrm{E}-06$ \\
\cline { 2 - 3 } & $0.1206 \mathrm{E}-09$ & $-0.6178 \mathrm{E}-09$ \\
\cline { 2 - 3 } & $-0.2554 \mathrm{E}-12$ & $0.6144 \mathrm{E}-10$ \\
\cline { 2 - 3 } & $0.2021 \mathrm{E}-15$ & $-0.1007 \mathrm{E}-11$ \\
\hline \multirow{4}{*}{$\mathbf{5 0}$} & $0.1250 \mathrm{E}-02$ & $0.2382 \mathrm{E}-02$ \\
\cline { 2 - 3 } & $-0.4865 \mathrm{E}-08$ & $-0.1925 \mathrm{E}-06$ \\
\cline { 2 - 3 } & $0.1858 \mathrm{E}-10$ & $0.4406 \mathrm{E}-08$ \\
\cline { 2 - 3 } & $-0.3022 \mathrm{E}-13$ & $-0.9584 \mathrm{E}-10$ \\
\cline { 2 - 3 } & $0.1676 \mathrm{E}-16$ & $0.7282 \mathrm{E}-12$ \\
\hline \multirow{4}{*}{60} & $0.1042 \mathrm{E}-02$ & $0.1984 \mathrm{E}-02$ \\
\cline { 2 - 3 } & $-0.3544 \mathrm{E}-08$ & $-0.7056 \mathrm{E}-07$ \\
\cline { 2 - 3 } & $0.9560 \mathrm{E}-11$ & $0.1844 \mathrm{E}-09$ \\
\cline { 2 - 3 } & $-0.1188 \mathrm{E}-13$ & $-0.2260 \mathrm{E}-11$ \\
\cline { 2 - 3 } & $0.5397 \mathrm{E}-17$ & $0.1068 \mathrm{E}-13$ \\
\hline
\end{tabular}

Table (3): Coefficients results of the mirrors, for $f_{1} / D_{1}=7, D_{2} / D_{1}=0.3$, and different values of $\mathrm{D}_{1}$

\begin{tabular}{|c|c|c|}
\hline \multirow{2}{*}{$\mathbf{D}_{\mathbf{1}}(\mathbf{c m})$} & $\begin{array}{c}\text { Primary } \\
\text { Mirror }\end{array}$ & Secondary Mirror \\
\hline \multirow{4}{*}{40} & $0.8925 \mathrm{E}-03$ & $0.1701 \mathrm{E}-02$ \\
\cline { 2 - 3 } & $0.8537 \mathrm{E}-08$ & $-0.2839 \mathrm{E}-07$ \\
\cline { 2 - 3 } & $-0.6890 \mathrm{E}-10$ & $0.3355 \mathrm{E}-09$ \\
\cline { 2 - 3 } & $0.2215 \mathrm{E}-12$ & $-0.5623 \mathrm{E}-10$ \\
\cline { 2 - 3 } & $-0.2458 \mathrm{E}-15$ & $0.1083 \mathrm{E}-11$ \\
\hline \multirow{4}{*}{$\mathbf{5 0}$} & $0.7142 \mathrm{E}-03$ & $0.1361 \mathrm{E}-02$ \\
\cline { 2 - 3 } & $0.1004 \mathrm{E}-08$ & $-0.3743 \mathrm{E}-07$ \\
\cline { 2 - 3 } & $-0.5613 \mathrm{E}-11$ & $0.4365 \mathrm{E}-09$ \\
\cline { 2 - 3 } & $0.1298 \mathrm{E}-13$ & $-0.5054 \mathrm{E}-11$ \\
\cline { 2 - 3 } & $-0.1044 \mathrm{E}-16$ & $0.2306 \mathrm{E}-13$ \\
\hline \multirow{4}{*}{60} & $0.5953 \mathrm{E}-03$ & $0.1134 \mathrm{E}-02$ \\
\cline { 2 - 3 } & $-0.1903 \mathrm{E}-09$ & $-0.8502 \mathrm{E}-08$ \\
\cline { 2 - 3 } & $0.1060 \mathrm{E}-11$ & $-0.2765 \mathrm{E}-09$ \\
\cline { 2 - 3 } & $-0.2339 \mathrm{E}-14$ & $0.6067 \mathrm{E}-11$ \\
\cline { 2 - 3 } & $0.1485 \mathrm{E}-17$ & $-0.4046 \mathrm{E}-13$ \\
\hline
\end{tabular}


From the previous tables, we notice the following:

When $\mathrm{D}_{1}$ increased, while $\mathrm{f}_{1} / \mathrm{D}_{1}$ ratio and $D_{2} / D_{1}$ ratio are constant; this will lead to decrease the coefficients values, of the two-mirrors. In addition, when $f_{1} / D_{1}$ ratio increased, while $D_{1}$ and $D_{2} / D_{1}$ ratio are constant; this will lead to decrease some of coefficients values of the two-mirrors.

\section{6- Rayleigh Limit (Rayleigh}

Criterion): It is used as a criterion for the selection of the dimensions, ratios and values, which displayed in this research. It is calculated according to the following relation [16]:

$$
\frac{2 \lambda \sqrt{p}}{n} \ngtr \frac{\lambda}{4} \ldots
$$

Where: $\lambda$ is the wavelength of the visible light $(550 \mathrm{~nm})$, and $P$ is the number of reflections from surfaces, which are good to $\frac{\lambda}{n}$. Therefore, $n=$ 11.31

Theoretical value of $\frac{\lambda}{n}=48.6 \mathrm{~nm}$

The theoretical value must be compared with the practical values, according to the following relation [16]:

$\Delta X_{\text {max. }} /$ or $\Delta X_{\text {max. }}^{\prime}>\frac{\lambda}{n}$

Where: $\Delta \mathrm{X}_{\max }$ is the maximum difference of the imitation value, from the actual value of the primary mirror, and $\Delta X_{\text {max. }}^{\prime}$ is the maximum difference of the imitation value, from the actual value of the secondary mirror.

Table (4), represents the results of the maximum difference between the actual and imitation values, of the mirrors $\Delta X_{\text {max. }}$ and $\Delta X_{\text {max. }}^{\prime}$, respectively, for $D_{1}=40,50$, and 60 $\mathrm{cm}, \mathrm{f}_{1} / \mathrm{D}_{1}=4,7, \mathrm{D}_{2} / \mathrm{D}_{1}=0.3$, and number of coefficients $=5$
Table (4): Results of the maximum difference between the actual and imitation values of the mirrors, at number of coefficients $=5$ and $D_{2} / D_{1}$ $=0.3$, for different values of $f_{1} / D_{1}$ and $D_{1}$

\begin{tabular}{|c|c|c|c|}
\hline $\mathbf{f}_{\mathbf{1}} / \mathbf{D}_{\mathbf{1}}$ & $\mathbf{D}_{\mathbf{1}}(\mathbf{c m})$ & $\Delta \mathbf{X}_{\max .}(\mathbf{c m})$ & $\Delta \mathbf{X}_{\text {max. }}^{\prime}(\mathbf{c m})$ \\
\hline \multirow{4}{*}{4} & 40 & 0.00010202 & 0.00001601 \\
\cline { 2 - 4 } & 50 & 0.00026456 & 0.00002662 \\
\cline { 2 - 4 } & 60 & 0.00009966 & 0.00002551 \\
\hline \multirow{2}{*}{$\mathbf{7}$} & 40 & 0.00001871 & 0.00001221 \\
\cline { 2 - 4 } & 50 & 0.00002874 & 0.00006655 \\
\cline { 2 - 4 } & 60 & 0.00003820 & 0.00001283 \\
\hline
\end{tabular}

The shaded values, shown in the previous table represent the best practical values of both $\Delta X_{\max }$. and $\Delta X_{\text {max.; }}^{\prime}$ which are compared with the theoretical value $(0.00000486 \mathrm{~cm})$.

\section{Conclusions:}

The number of the coefficients increased, i.e., increasing the terms of the equation of the primary and secondary mirrors (polynomial function), this will lead to decrease the difference between the actual and imitation values of the two-mirror profile. Therefore, this will lead to increase the accuracy of the results.

The Aplanatic Cassegrain Telescope (ACT) system, gives good results, within primary mirror diameter, $\mathbf{D}_{\mathbf{1}}=\mathbf{4 0} \mathrm{cm}$. In addition, focal ratio of the primary mirror, $\mathbf{f}_{\mathbf{1}} / \mathbf{D}_{\mathbf{1}}=\mathbf{7}$. Finally, the obscuration ratio of the secondary mirror, $\mathbf{D}_{2} / \mathbf{D}_{\mathbf{1}}=\mathbf{0 . 3}$

\section{References:}

1. Ahmed, A. K. 2009. "Determination of Profiles of Cassegrain Telescope Mirrors", Publish in Proceeding of $3^{\text {rd }}$ scientific conference of the College of Science, University of Baghdad, 24-26 March, PP. 714-723.

2. Fix, J. D. 2006. "Astronomy (Journey to the Cosmic Frontier),", The McGraw-Hill Companies, Inc.
3. Ahmed, A. $\mathrm{K}$. 2002. "Determination of Profiles of 
Gregorian Telescope Mirrors", M.Sc. Thesis, College of Science, University of Baghdad.

4. Jastrow, R. and Thompson, M. H. 1977. "Astronomy (Fundamentals and Frontiers)", Robert Jastrow, $3^{\text {rd }}$ edition.

5. Ali, S. M. 2001. "Optical Design Study of a Cassegrain Telescope", M.Sc. Thesis, College of Science, University of Baghdad.

6. Macdonald, J. 1977. "Second European Optics Summer School", University of Reading.

7. Wolf, E. 1988. "Progress in Optics", North-Holand, V. XXV.

8. Shin, I. F., Michelson, L. and Chang, D. B. 1985. "Aplanatic Two-Mirror Compact Collimator", SPIE, V. 554, 265-271.

9. Conrady, A. E. 1957. "Applied Optics and Optical Design”, Dover Publications, Inc., Part One.

10. Rosenhauer, K. 1967. "Advanced Optical Techniques”, edited by A. C. S. Vanheel, North-Holland Publishing Co.
11. Tatian, B. 1985. "Testing an Unusual Optical Surface”, SPIE, V. 554, 139-147.

12. Camacho, A. A. and Solano, D. C. 1999. "Application of Aspherical Surfaces in Optics", Revista Mexicana de Fisica, 45(3), 315321.

13. Wassermann, G. D. and Wolf, E. 1949. "On the Theory of Aplanatic Aspheric Systems", Proc. Phys. Soc., 62(349), 2-8.

14. Kuo, S. S. 1972. "Computer Applications of Numerical Methods", $\quad$ Addison-Wesley Publishing Company, Inc.

15. Sidgwick, J. B. 1980. "Amateur Astronomer's Handbook", Enslow Publishers, $4^{\text {th }}$ edition.

16. Ahmed, M. S. 1987. "Optical Design Study of a New Type of Compact Astronomical Telescope", Ph.D. Thesis, University of Manchester.
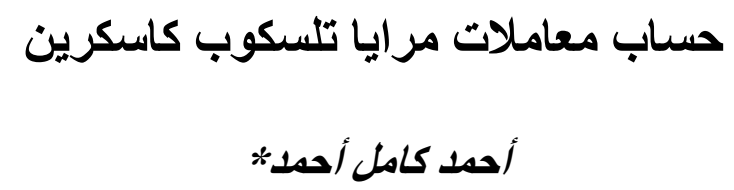

*قمم الفلك ـ كلية العلوم - جامعة بغداد

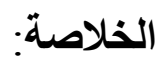

تم في هذا البحث, بناء برنامج لحساب معاملات منظومة تلسكوب كاسكرين اللازيغي, خالياً من نأثنير

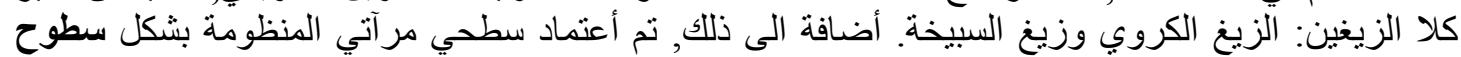

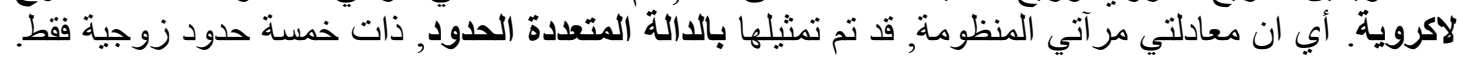

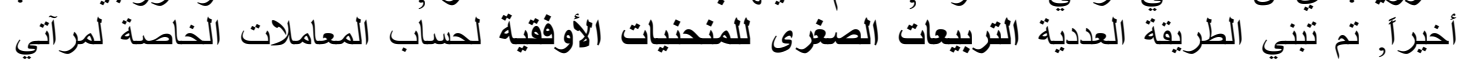

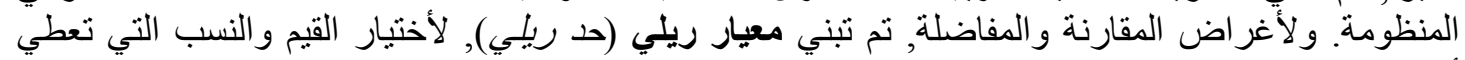
أفضل النتائج. 